\title{
Anesthetic management of cesarean section of a wheelchair bound parturient with myelomeningocele
}

\author{
Shankar Lal, Umair Akram, Tharwat Aisa, Adil Sher, Zulfiqar Memon, Rose Mary Moriarty
}

Correspondence: Dr. Shankar Lal, Our Lady of Lourdes Hospital, Drogheda, Ireland; E-mail: shankar.anaesthesia1@gmail.com

\section{Abstract}

Neural tube defects (NTD) affect 1-2 in 1000 pregnancies over the world. The most frequent spinal dysraphisms are meningocele and myelomeningocele while the spina bifida occulta, tethered cord, lipomyelomeningocele, split cord malformations, neuroenteric cyst, and several other conditions occur less frequently. Open spinal dysraphisms (meningocele and myelomeningocele) are compatible with postnatal survival, but they can result in severe neurologic impairment as inability to ambulate, urinary incontinence, hydrocephalus, scoliosis, and gastrointestinal (GI) disorders. Closed spinal dysraphisms (spina bifida occulta, tethered cord, lipomyelomeningocele, split cord malformations, neuroenteric cyst) are often less severe and sometimes asymptomatic but may result in severe neurologic impairment secondary to spinal cord tethering. Modern health care facilities has resulted in a significant decrease in the mortality rate in patients with SB2 along with improved survival rate up to $80 \%$. Due to the neurologic complexity and severity of these conditions, it is paramount to understand the challenges and offer the best anesthetic approach for these patients. To the best of our knowledge, this is the first case of SB going under caesarean delivery in our hospital. We wish to highlight our experience of perioperative management of this patient.

Key words: Spina bifida, anesthetic management

Citation: Lal S, Aisa T, Akram U, Sher A, Memon Z, Moriarty RM. Anesthetic management of cesarean section of a wheelchair bound parturient with myelomeningocele. Anaesth. pain intensive care 2020;24(5):565-567.

Received: 13 June 2020, Reviewed: 24 June 2020, Revised: 18 July 2020, Accepted: 8 August 2020

\section{Introduction}

Neural tube defects (NTD) affect 1-2 in 1000 pregnancies over the world. The most frequent spinal dysraphisms are meningocele and myelomeningocele while the spina bifida occulta, tethered cord, lipomyelomeningocele, split cord malformations, neuroenteric cyst, and several other conditions occur less frequently. ${ }^{1}$ Open spinal dysraphisms (meningocele and myelomeningocele) are compatible with postnatal survival, but they can result in severe neurologic impairment as inability to ambulate, urinary incontinence, hydrocephalus, scoliosis, and gastrointestinal (GI) disorders. Closed spinal dysraphisms (spina bifida occulta, tethered cord, lipomyelomeningocele, split cord malformations, neuroenteric cyst) are often less severe and sometimes asymptomatic but may result in severe neurologic impairment secondary to spinal cord tethering. ${ }^{2}$ Modern health care facilities has resulted in a significant decrease in the mortality rate in patients with $\mathrm{SB}^{2}$ along with improved survival rate up to $80 \%$. $^{3}$

Due to the neurologic complexity and severity of these conditions, it is paramount to understand the challenges and offer the best anesthetic approach for these patients.

To the best of our knowledge, this is the first case of SB going under cesarean delivery in our hospital. We wish to highlight our experience of perioperative management of this patient.

\section{Case report}

We present a 29-year-old primigravida with history of SB admitted in our hospital for scheduled cesarean section. She was born with myelomeningocele, and had a corrective surgery on the $3^{\text {rd }}$ day of life. Later her course of development was complicated by obstructive hydrocephalus and neurogenic bladder resulting in significant limitation of mobility and recurrent urinary tract infections and required insertion of suprapubic 
catheter and insertion of VP shunt at the age of 12 years, respectively.

She was assessed by our anesthetic team in the clinic and her examination revealed

High BMI of over 40, severe deformity of both legs with massive swelling of the left side increasing the risk of DVTs. She had a history of recurrent urinary tract infections. Her SPC was changed every three months and she was on nitrofurantoin for long term. No neurological deficit was found in the upper limbs. Her airway examination showed short neck, increased neck circumference, limited neck extension and Mallampati score of 3. Chest X-ray showed reduced lung volumes due to her posture. Other vitals were normal. Multidisciplinary team meetings were arranged between anesthesia, obstetric and urology teams and a plan was put in for elective cesarean section at $38+$ weeks of gestation. Management plan was discussed with the patient and agreed. She had been receiving her routine medicine supplemented with folic acid throughout pregnancy with regular monitoring of blood levels. Antenatal checkups and ultrasound for fetal wellbeing were performed regularly.

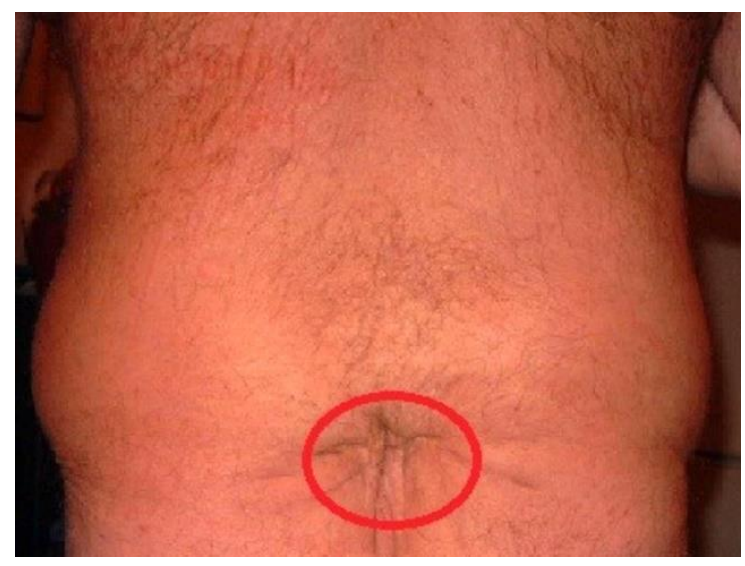

\subsection{Anesthetic management}

As a potential difficult airway due to factors mentioned above, we ensured rapid and safe intubation by the rapid sequence induction with video laryngoscope. Oxford pillow was used for head elevation. Patient was pre-oxygenated for three minutes to achieve $\mathrm{EtO}_{2}$ levels over $90 \%$. We used propofol and suxamethonium for induction. Intubation was easy and ETT position was confirmed by bilateral chest rise, auscultation and capnography. She remained hemodynamically stable after induction and intraoperatively requiring no vasopressors. Muscle relaxation was maintained with rocuronium. IV paracetamol and morphine were given for analgesia intraoperatively. Midline vertical incision was chosen by the obstetrician due to technical challenges posed by high BMI, indwelling SPC and difficulty in positioning of patient.

Baby girl was born and was received by the pediatric team in the theatre. Total blood loss was about $350 \mathrm{mls}$. Post-operative analgesia was managed with oral opioids, paracetamol and Ibuprofen along with prophylactic LMWH to prevent DVTs.

Baby girl was examined by pediatric team and found to have anal atresia with no other signs of any neurological and musculoskeletal defects. At the end of surgery SPC was changed by the Urologist. Extubation was uneventful and patient was safely transferred to post-operative care unit. On day 5th of C/Section patient was discharged home with the advice given to contact hospital in case of any postoperative problems.

\section{Discussion}

Due to the rare presentation of the patient with spina bifida for cesarean section, we wished to highlight our strategic approach towards the management of the patient going for elective cesarean section in our hospital. For early detection of any neural tube defects and fetal wellbeing, our patient underwent regular screenings, counselling and lab tests. Due to the neurogenic bladder and suprapubic catheter, her antenatal period was complicated by frequent urinary tract infections. Meticulous care and timely change of suprapubic catheter was performed to prevent the recurrence of UTIs.

Patients with SB have an increased predisposition to preterm labour ${ }^{4}$ possibly related to urinary tract infections or undiagnosed coexisting genital tract abnormalities such as bicornuate uterus. There are case reports of safe vaginal deliveries among patients with $\mathrm{SB},{ }^{5}$ but we planned elective $\mathrm{CS}$ at $38+$ weeks as the most appropriate and safe mode of delivery for our patient because of her high risk factors i.e. high BMI, indwelling suprapubic catheter and previous spinal surgery aiming to avoid potential prolonged labor specially second stage of labor which could be challenging and emergency cesarean 
section. Establishing neuraxial anesthesia in patients with SB is challenging due to spinal cord deformities, back surgeries old scars and fibrosis which could result in cord injury. ${ }^{6-7}$ We preferred general anesthesia in our patient to avoid all the complications.

Timely management, proper counselling and multidisciplinary discussion among obstetrician, and anesthetist resulted in a good outcome of our patient.

\section{Conclusion}

Due to the advanced care and early interventions, patients with SB are surviving to adulthood. Female patients may present to the maternity units, thus to be familiar with obstetric, urologic, surgical, and anesthetic challenges in the perioperative care of such patients will be beneficial to improve outcomes and reduce the risk of complications.

\section{Conflicts of interest}

None declared by the authors.

\section{Authors' contribution}

SL: Writing, Literature search and drafting

UA, TA, AS: Literature search

ZM, RMM: Supervision in writing

\section{References}

1. Schoner K, Axt-Fliedner R, Bald R, Fritz B, Kohlhase J, Kohl T, et al. Fetal Pathology of Neural Tube Defects An Overview of 68 Cases. Geburtshilfe Frauenheilkd. 2017 May;77(5):495-507. [PubMed] DOI: 10.1055/s0043-103459

2. Heuer GG, Moldenhauer JS, Scott Adzick N. Prenatal surgery for myelomeningocele: review of the literature and future directions. Childs Nerv Syst. 2017 Jul;33(7):1149-1155. [PubMed] DOI: 10.1007/s00381017-3440-z

3. Meller $\mathrm{C}$, Aiello H, Otaño L. Sonographic detection of open spina bifida in the first trimester: review of the literature. Childs Nerv Syst. 2017 Jul;33(7):1101-1106. [PubMed] DOI: 10.1007/s00381-017-3443-9

4. Rietberg CC, Lindhout D. Adult patients with spina bifida cystica: genetic counselling, pregnancy and delivery. Eur J Obstet Gynecol Reprod Biol. 1993 Nov;52(1):63-70. [PubMed] DOI: 10.1016/00282243(93)90227-4

5. Arata M, Grover S, Dunne K, Bryan D. Pregnancy outcome and complications in women with spina bifida. J Reprod Med. 2000 Sep;45(9):743-748. [PubMed]

6. Crosby ET, Halpern SH. Obstetric epidural anaesthesia in patients with Harrington instrumentation. Can J Anaesth. 1989 Nov;36(6):693-696. [PubMed] DOI: 10.1007/BF03005423

7. Vaagenes $P$, Fjaerestad I. Epidural block during labour in a patient with spina bifida cystica. Anaesthesia. 1981 Mar;36(3):299-301. [PubMed] DOI: 10.1111/j.13652044.1981.tb10205.x 